\title{
Reabilitação estética anterior com sistema IPS e.max: relato de caso
}

\author{
Anterior aesthetic rehabilitation with IPS e.max system: case report \\ Rehabilitación estética anterior con el sistema IPS e.max: reporte de caso
}

\author{
Ithalo Lessa EBERLE \\ Gisely Naura VENÂNCIO \\ Everaldo Pereira de AQUINO3 \\ Carolina Rocha AUGUSTO

\begin{abstract}
${ }^{1}$ Graduado em Odontologia, Faculdade de Odontologia, Universidade Nilton Lins, UNINILTON LINS, Avenida Professor Nilton Lins, 3259. Parque das Laranjeiras. CEP 69058-030. Manaus-AM ${ }^{2}$ Departamento de Dentística e Prótese, Faculdade de Odontologia, Universidade Nilton Lins, UNINILTON LINS, Avenida Professor Nilton Lins, 3259. Parque das Laranjeiras. CEP 69058-030. Manaus-AM

${ }^{3}$ Departamento de Endodontia, Faculdade de Odontologia, Universidade Nilton Lins, UNINILTON LINS, Avenida Professor Nilton Lins, 3259. Parque das Laranjeiras. CEP 69058-030. Manaus-AM
\end{abstract}

\section{Resumo}

A utilização de coroas livres de metal em dentes anteriores tem sido amplamente utilizada nos casos de reabilitação estética. Os sistemas cerâmicos modernos apresentam alta estabilidade de cor, resistência ao desgaste, estética semelhante aos dentes naturais e grande índice de sucesso clínico, se bem executado. O objetivo deste trabalho foi relatar um caso clínico de reabilitação estética em dentes anteriores superiores utilizando coroas totalmente cerâmicas associadas a retentores intrarradiculares. O plano de tratamento adotado para o caso foi a remoção dos núcleos antigos, retratamento endodôntico dos elementos, cimentação de novos retentores, gengivoplastia e cimentação de coroas unitárias IPS e.max. O tratamento proposto permitiu reestabelecer estética, função e devolução da autoestima, relatada pela paciente. A reabilitação anterior com sistema IPS e.max pode ser uma alternativa que reúne qualidades estéticas com excelentes propriedades mecânicas.

Descritores: Estética; Prótese Dentária; Porcelana Dentária.

\begin{abstract}
The use of metal free crowns on anterior teeth have been widely used in cosmetic rehabilitation cases. Modern ceramic systems have high color stability, wear resistance, aesthetic similar to natural teeth and large clinical success rate, if well executed. The aim of this study was to report a case of aesthetic rehabilitation in upper front teeth with all-ceramic crowns associated with intraradical retainers. The adopted treatment plan for the event was the removal of the old cores, endodontic retreatment of the elements, cementing new retainers, gingivoplasty and cementation of crowns IPS e.max. The proposed treatment allowed restore aesthetics, function and return self-esteem, reported by the patient. The previous rehabilitation with IPS e.max can be an alternative that combines aesthetic qualities with excellent mechanical properties.
\end{abstract}

Descriptors: Esthetics; Dental Prosthesis; Dental Porcelain.

\section{Resumen}

El uso de coronas libres de metal en los dientes anteriores han sido ampliamente utilizados en casos de rehabilitación cosmética. Sistemas cerámicos modernos tienen una alta estabilidad de color, resistencia al desgaste, estética similar a los dientes naturales y una gran tasa de éxito clínico, si bien ejecutada. El objetivo de este estudio fue presentar un caso de la rehabilitación estética de los dientes delanteros superiores con coronas de cerámica asociados con pernos muñones. El plan de tratamiento aprobado para el evento fue la eliminación de los viejos núcleos, un nuevo tratamiento endodóntico de los elementos, consolidando nuevos pernos, gingivoplastia y cementación de coronas IPS e.max. El tratamiento propuesto permitió restaurar la estética, función y devolver la autoestima, comunicada por la paciente. La rehabilitación anterior con IPS e.max puede ser una alternativa que combina cualidades estéticas con excelentes propiedades mecânicas.

Descriptores: Estética; Prótesis Dental; Porcelana Dental. 


\section{INTRODUÇÃO}

A insatisfação estética é um dos relatos mais comuns na clínica odontológica diária, bem como perda de função e falta de conforto ${ }^{1}$. Restaurações diretas e indiretas insatisfatórias, alteração da forma, cor, tamanho e injúrias periodontais afetam tanto a saúde como a estética do paciente, prejudicando sua autoestima e qualidade de vida ${ }^{2}$. Para reestabelecer a estética, o cirurgião-dentista deve avaliar desde a saúde periodontal até as condições dentais e protéticas. Um dos fatores essenciais para obter sucesso na estética dentro da área da prótese fixa é o planejamento que será obtido através do diálogo entre o profissional e paciente em relação às expectativas do tratamento $^{3}$. Uma reabilitação estética deve ser indicada baseando-se em fatores como a destruição da estrutura dental, estética comprometida, controle de biofilme, considerações de custo e retenção ${ }^{4}$.

A reabilitação protética dos elementos dentários por meio de prótese fixa visa devolver a integridade das arcadas, restabelecendo as funções normais como mastigação, deglutição e fonação, evitando a instalação de hábitos parafuncionais e má-oclusões. Além disso, devolve a estética, fator importante para o bem estar do paciente ${ }^{5}$. Em regiões anteriores onde se busca harmonia, as próteses livres de metal passaram a ter maior índice de sucesso devido ao desenvolvimento de novos sistemas cerâmicos para próteses unitárias, com propriedades mecânicas e físicas semelhantes às das ligas metálicas e que atendem também ao requisito estético ${ }^{6}$. Diversos tipos de sistemas cerâmicos estão disponíveis atualmente para confecção de coroas unitárias sem metal, demonstrando valores de resistência semelhantes ou superior às porcelanas convencionais. Estes sistemas podem ser baseados em óxido de silício, alumizadas ou alumizadas reforçadas por óxido de zircônia ${ }^{6}$. O sistema cerâmico livre de metal IPS e.max possui a capacidade de reproduzir a naturalidade da estrutura dentária e apresenta quatro materiais altamente estéticos e resistentes que torna o sistema flexível para o técnico em prótese 7 .

A abordagem multidisciplinar nos casos de reabilitação é necessária para obter sucesso variando de acordo com a complexidade do tratamento. Quando a queixa principal está associada ao descontrole do estado de saúde geral do paciente, como doença periodontal, o prognóstico de uma etapa depende do sucesso de outra, se fazendo necessária a abordagem multidisciplinar. Um planejamento que visa interdisciplinaridade aumenta a chance de sucesso no tratamento, aperfeiçoa a atuação profissional e integra as áreas como endodontia, periodontia e prótese para o preparo bucal em diferentes planejamentos ${ }^{8}$.

O objetivo deste trabalho foi descrever um relato de caso de reabilitação estética anterior superior com sistema cerâmico IPS e.max com foco no planejamento multidisciplinar para garantir funcionalidade, saúde periodontal e estética.

\section{CASO CLÍNICO}

Paciente de 45 anos de idade, gênero feminino, procurou atendimento na clínica odontológica da Universidade Nilton Lins apresentando queixa principal de insatisfação estética nos incisivos superiores. Na anamnese, apresentou bom estado geral de saúde. Sua história odontológica pregressa consta tratamento endodôntico nos elementos 11, 21 e 22 há aproximadamente 29 anos, seguida de instalação de coroas unitárias. No exame clínico intra-oral foram observadas coroas metalocerâmicas com estética insatisfatória (Figura 1), associadas a núcleos metálico fundidos (NMF) nos elementos 11, 21 e 22. A margem gengival apresentava-se escurecida, com contornos e zênites não harmoniosos no sextante S2. O elemento dentário 12 possuía uma restauração em resina composta classe III, com infiltração e lesão cariosa ativa. Após o exame radiográfico, foi observada lesão periapical nos elementos dentários 12 , 21 e 22, e obturação endodôntica insatisfatória no elemento 11 (Figura 2).

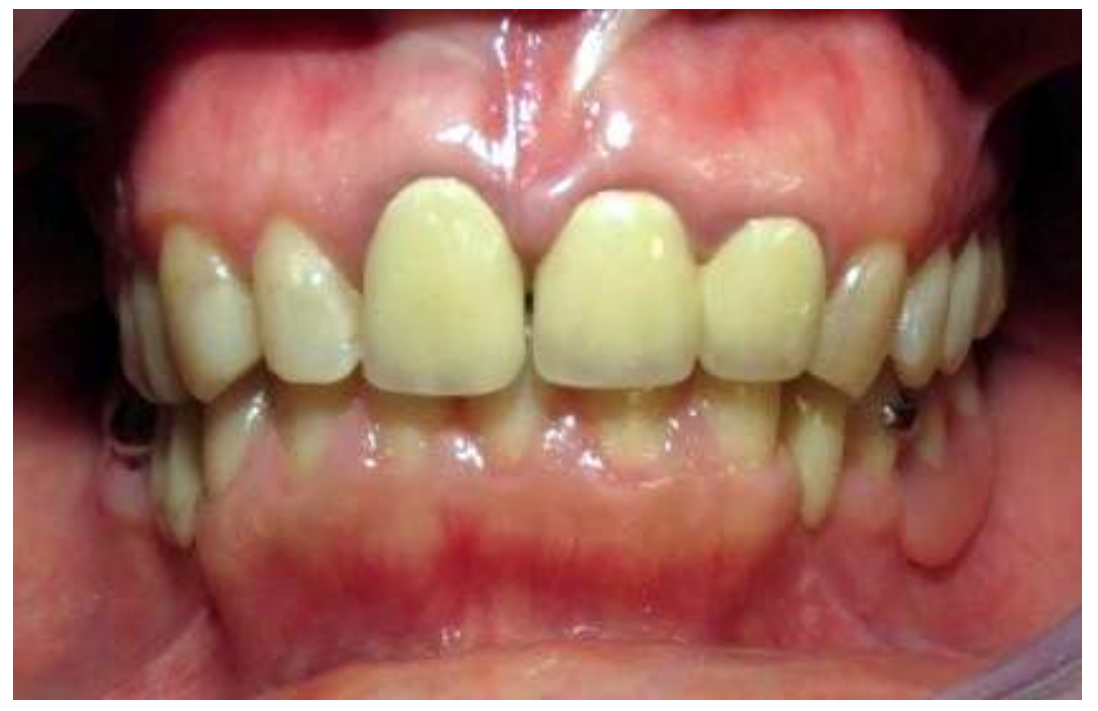

Figura 1. Aspecto clínico inicial

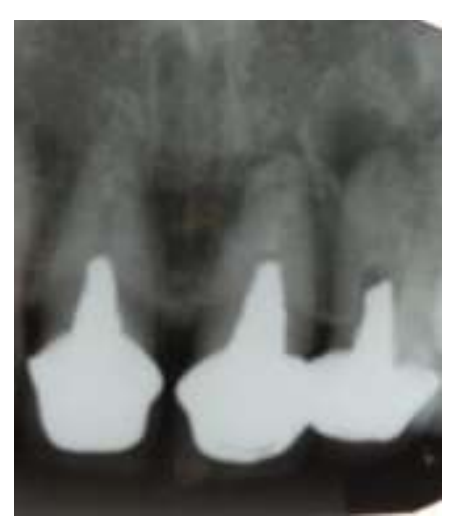

Figura 2. Radiografia inicial dos elementos 12, 21 e 22

O planejamento baseou-se na remoção dos NMF, tratamento/ retratamento endodôntico dos elementos citados, gengivoplastia para correção do formato e escurecimento gengival na região dos elementos 14 ao 24 , cimentação de retentor 
intrarradicular e instalação de coroas unitárias. Inicialmente foi realizado tratamento endodôntico do elemento 12 pela técnica de instrumentação coroaápice. $\mathrm{Na}$ consulta seguinte, foram removidas as coroas e NMF. As coroas provisórias foram confeccionadas pela técnica direta com dentes de estoque cor 69, com retenção intrarradicular com fio ortodôntico 0,6mm (Figura 3). Os elementos foram instrumentados e medicados. Após 20 dias, com objetivo de alcançar um amplo efeito terapêutico na região periapical a paciente retornou sem sintomatologia e a obturação dos canais radiculares foi realizada.

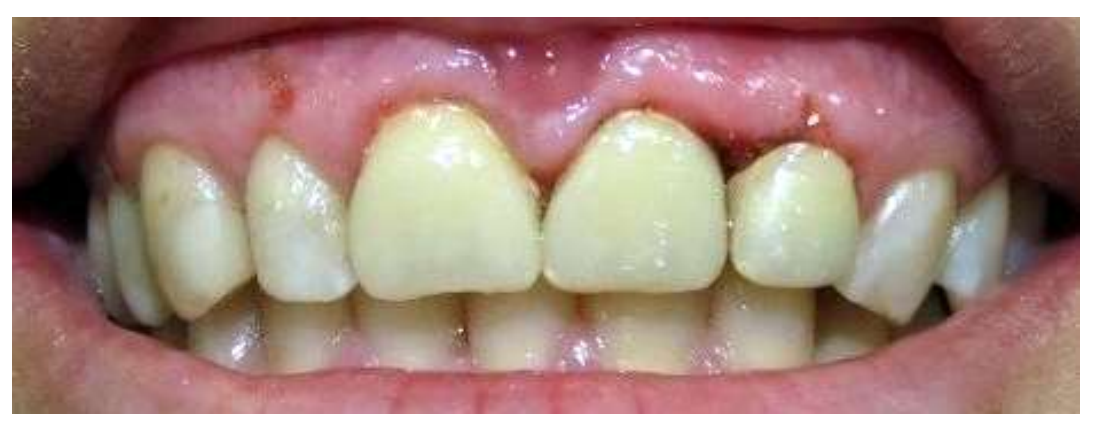

Figura 3. Restaurações provisórias nos elementos 11, 21 e 22

Considerando os critérios de comprimento do pino ${ }^{9}$, os canais foram desobturados e preparados com auxílio das brocas Gates-Glidden e Largo. Os condutos dos elementos 11, 21 e 22 foram moldados pela técnica direta com resina acrílica para confecção dos NMF. No elemento 12, por haver efeito férula ${ }^{10}, \mathrm{o}$ retentor escolhido foi pino de fibra de vidro $\mathrm{n}^{\circ} 0.5$ (Exacto Post, Angelus, Brasil). Após a cimentação dos retentores (Figura 4), a cirurgia gengival foi realizada para correção dos zênites e contornos (Figura 5) na região vestibular dos elementos 14, 13, 12, 11, 21, 22, 23 e 24 . Após o período de cicatrização de 21 dias ${ }^{10}$ os preparos foram reajustados conforme a nova margem gengival obtida, com términos $0,5 \mathrm{~mm}$ subgengival ${ }^{11}$.

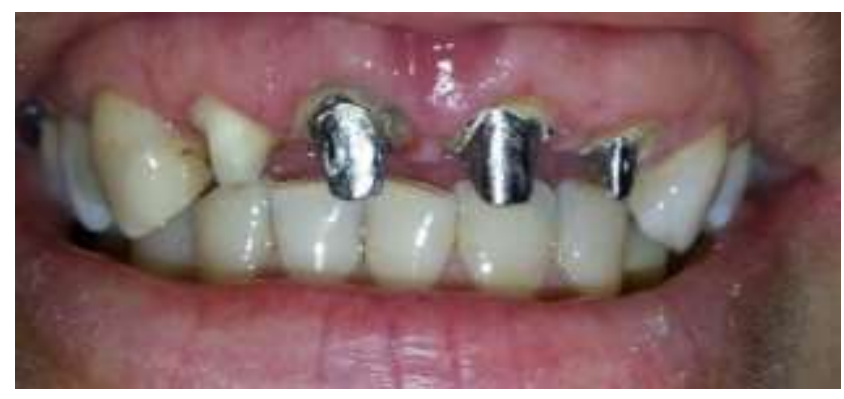

Figura 4. NMF cimentados

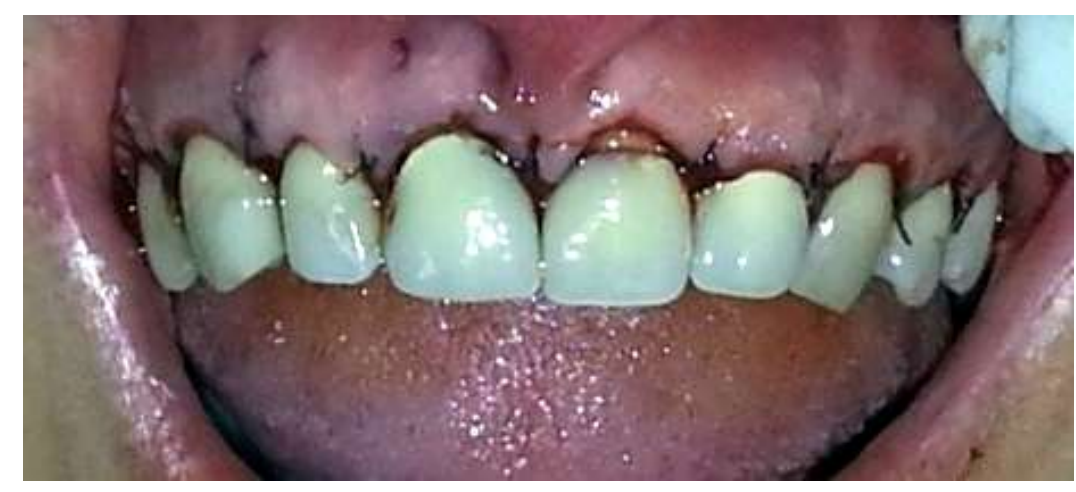

Figura 5. Aspecto clínico após gengivoplastia
A moldagem, pela técnica de dupla impressão, foi realizada com auxílio de fio retrator e silicone de condensação (Precise, Dentsply, EUA). Os modelos, confeccionados em gesso especial, foram troquelados para confecção das coroas definitivas no sistema IPS e.max ZirPress. O condicionamento das peças protéticas foi realizado segundo recomendações do fabricante com ácido fluorídrico a $10 \%$ por 20 segundos seguido de silanização. Os preparos foram condicionados com ácido fosfórico $37 \%$ por 15 segundos em dentina e 30 segundos em esmalte, lavados abundantemente e aplicado o sistema adesivo (Single Bond, 3M, ESPE, Brasil) conforme recomendações do fabricante. A cimentação das peças foi realizada sob isolamento relativo com inserção do cimento resinoso dual (AllCem, FGM, Brasil) no interior das peças e sobre os preparos, a fotoativação foi realizada por 40 segundos em cada face (Figura 6). Após remoção dos excessos com auxílio de lâmina de bisturi $\mathrm{n}^{\circ} 11$ foi realizado ajuste oclusal e orientações de higiene bucal.

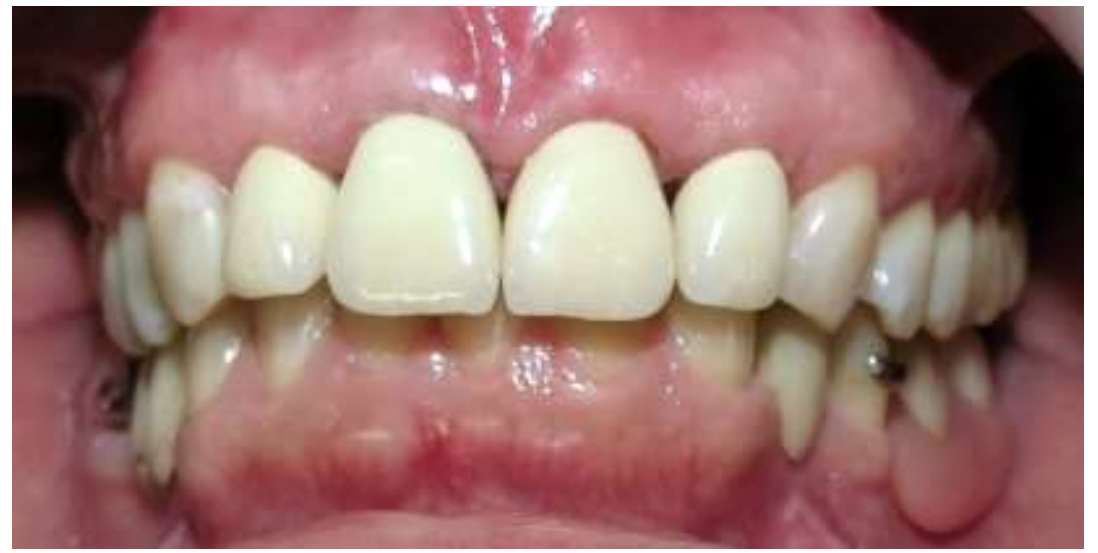

Figura 6. Aspecto final após cimentação das coroas definitivas

\section{DISCUSSÃO}

A indicação da prótese fixa proporciona desde a restauração de apenas um elemento até a reabilitação de toda arcada dentária ${ }^{4}$. Em relação ao material restaurador a ser utilizado em dentes anteriores, a literatura aponta dados concordantes de que a coroa metalocerâmica não é a melhor indicação pela impenetrabilidade da luz através da restauração, devido à infraestrutura metálica ${ }^{2,4,8}$.

Para reestabelecer a estética, sem deixar de lado a resistência e longevidade da prótese, é necessária indicação correta dos retentores intrarradiculares, que podem ser confeccionados com vários materiais e técnicas como núcleos metálicos fundidos ou pinos pré-fabricados dependendo do grau de destruição da coroa, do dente envolvido, do suporte ósseo, do tipo de prótese e dos tipos de forças às quais esses dentes serão submetidos ${ }^{13}$. No caso apresentado os elementos 11,21 e 22 apresentavam grande perda de estrutura coronária, já o elemento 12 possuía remanescente coronário acima de $50 \%$ da coroa clínica ${ }^{10}$. Em dentes com ampla destruição da coroa e uma morfologia 
radicular favorável, são frequentemente indicados os núcleos metálicos fundidos que possuem vantagens como larga experiência clínica, alta resistência e excelente radiopacidade, em contrapartida possui deficiência na estética, possibilidade de corrosão e alto módulo de elasticidade ${ }^{14}$. Já os pinos pré-fabricados, como o de fibra de vidro, apresentam além da estética vantagens como facilidade de confecção, menor custo e módulo de elasticidade semelhante à estrutura dental $^{15}$. Muniz et al. ${ }^{10}$ (2010) destacaram vantagens tais como menor tempo de execução clínica, melhor estética, preservação dos tecidos radiculares por não necessitar de preparo expulsivo como núcleos metálicos fundidos, porém é necessário que haja uma quantidade mínima de remanescente dental que seja acima de $2 \mathrm{~mm}$, chamado de férula, para conferir um efeito estabilizante do pino e obter melhor resistência à fratura.

As restaurações metalocerâmicas combinam resistência e precisão de um metal fundido com a estética da porcelana ${ }^{4}$. Porém, uma prótese unitária metalocerâmica não é escolha ideal para se restaurar dentes superiores anteriores quando a questão estética é a queixa principal. As próteses unitárias cerâmicas oferecem um grande percentual de sucesso em se assemelhar a aparência do dente natural adjacente ${ }^{16}$. A indicação das coroas unitárias totalmente cerâmicas tem como objetivo reabilitar principalmente a estética, autoestima e bem estar além de promover a saúde periodontal, periapical e melhorar a função mastigatória da paciente. Estudos recentes sugerem que a confecção de coroas com sistemas cerâmicos totalmente livres de metal são uma excelente alternativa restauradora, com potencial estético superior ao das coroas metalocerâmicas 2, 6, 17, 19, 20 .

Vários estudos mostram que a prótese de cerâmica pura é indicada em áreas onde se faz necessária cobertura total e estética máxima, no entanto não é indicada para reabilitação em dentes posteriores devido à maior tensão mastigatória ${ }^{4,}$, 8 . Outros estudos apontam que sistemas compostos por óxido de silício e óxido de zircônia, como o sistema IPS e.max, apresenta alta resistência flexural, sendo indicado para a confecção de coroas de cerâmica puras unitárias tanto anterior como posterior, inlays, onlays e facetas ${ }^{18}$.

O sistema IPS e.max é feito de uma composição versátil podendo ser de cerâmicas de vidro com base de dissilicato de lítio injetado ou fresado, como e.max Press e e.max CAD, ou óxido de zircônia injetado ou fresado, como e.max ZirPress e e.max $\mathrm{ZirCAD}^{7}$. O IPS e.max é preciso, pela técnica de prensagem das pastilhas que tem diversos níveis de opacidade para mascarar núcleos metálicos ${ }^{18}$. Pode ser utilizada na confecção de facetas, laminados, coroas de dentes posteriores, pontes fixas anteriores e posteriores até pré-molares dentre outras indicações ${ }^{19}$. A adaptação marginal tem aceitação clínica com valores abaixo de $120 \mu \mathrm{m}$, preenchendo os requisitos de longevidade ${ }^{7}$.

Todos os sistemas e.max possuem ótimos resultados estéticos, porém a melhor adaptação marginal é daqueles que utilizam o sistema CAD. Em relação à resistência, o sistema e.max Press tem se mostrado superior ao e.max CAD tendo maiores possibilidades de indicações, porém, o sistema de maior resistência flexural, com $900 \mathrm{Mpa}$, é o e.max ZirCAD, que pode ser utilizado em todas as situações clínicas. O sistema e.max ZirPress tem maior grau de opacidade, contribuindo para um bom resultado estético, podendo ser utilizado em casos em que seja necessário opacificar um NMF. Devido sua versatilidade, o sistema e.Max preenche requisitos mecânicos e estéticos, desde que seja feita uma indicação precisa para cada caso, além de não burlar as etapas de cimentação ${ }^{20}$. No caso apresentado, optou-se pelo sistema e.Max ZirPress devido sua excelente capacidade de mascarar o retentor metálico, mantendo assim o excelente padrão estético.

Outra etapa decisiva para o sucesso da longevidade de uma reabilitação é a saúde periodontal. A periodontia é uma área de estreita relação com a prótese fixa, pois vários fatores estão relacionados à saúde periodontal, como forma, contorno, higiene oral e localização da margem cervical do preparo ${ }^{3}$. A inflamação periodontal ocorre através do acúmulo de biofilme na margem gengival, então é necessário o condicionamento periodontal antes da instalação das próteses definitivas, para que não se instale doenças periodontais e ocorra infiltrações que levarão ao insucesso da reabilitação ${ }^{21}$. A reabilitação estética descrita neste relato, fundamentada no diagnóstico, planejamento e execução com uso do sistema IPS e.max ZirPress proporcionou um resultado estético e funcional satisfatório, atendendo as expectativas da paciente.

\section{CONCLUSÃO}

A reabilitação estética com coroas livres de metal de IPS e.max obteve excelente resultado estético e funcional no caso apresentado. Para o sucesso do tratamento, a abordagem multidisciplinar foi determinante, visto que a etapa de reabilitação protética dependeu de outras áreas como endodontia e periodontia.

\section{REFERENCIAS}

1. Mori TA, expectativas com relação aos resultados estéticos dos tratamentos odontológicos, [dissertação]. São Paulo: Universidade de São Paulo; 2003.

2. Rossato D.M, Saade E.G, Saad J.R.C, Porto-Neto S.T. Coroas estéticas anteriores em cerâmica 
metalfree: relato de caso clínico. Rev Sul-Bras Odontol. 2010;7(4):494-8.

3. Pegoraro L.F, Valle A.L, Araújo C.R.P, Bonfante G, Rodrigues P.C, Bonachela V. Prótese Fixa. São Paulo: Artes Médicas; 1998.

4. Shillingburg H.T. Fundamentos de prótese fixa. $4^{\mathrm{a}}$ ed. São Paulo: Quintessence; 2007.

5. Brelaz K.L.A.T. Prótese provisória estética: relato de caso. [trabalho de conclusão de curso]. Manaus: Universidade Nilton Lins; 2014.

6. Parreira G.G, Santos LM. Cerâmicas Odontológicas: Conceitos e Técnicas. São Paulo: Livraria Santos; 2005.

7. Clavijo VGR, Souza NC, Andrade MF. IPS e.Max: Harmonização do sorriso. Rev Dental Press Estét. 2007;4(1):33-49.

8. Soares MS, Morea C, Romano MM, Adde CA, Dominguez GC. Abordagem interdisciplinar em reabilitação bucal. Rev Assoc Paul Cir Dent. 2012; 66(4):260-7.

9. Bispo LB. Reconstrução de dentes tratados endodonticamente: retentores intra-radiculares: Revisão de literatura. RGO. 2008; 56(1):81-4.

10. Muniz L. Reabilitação estética em dentes tratados endodonticamente: pinos e possibilidades clínicas conservadoras. Leonardo Muniz e colaboradores, editores. São Paulo: Santos; 2010.

11. Damante CA. Avaliação clínica e histológica dos efeitos do laser em baixa intensidade na cicatrização de gengivoplastia em humanos [dissertação]. Bauru: Faculdade de Odontologia de Bauru; 2003.

12. Elias MG, Carvalho W, Barboza EP. Localização da margem dos preparos em restaurações estéticas: uma discussão em relação á saúde periodontal. RGO. 2013;61(12):441-5.

13. Sigemori RM, Prieto LT, Oliveira DCRS, Coppini EK, Araújo CTP, Dias CTS, Paulillo LAMS. Reforço intrarradicular de raízes debilitadas. Rev Bras Odontol. 2012;69(2):250-4.

14. Moro M, Agostinho AM, Matsumoto W. Núcleos metálicos fundidos x pinos pré-fabricados. PCL, 2005; 7(36): 167-72.

15. Niehues LP. Cimentação de pinos de fibra: Revisão de literatura. [trabalho de conclusão do curso]. Florianópolis: Universidade Federal de Santa Catarina; 2012.

16. Anusavice KJ. Phillips: Materiais Dentários. $11^{\mathrm{a}}$ ed. Rio de Janeiro: Elsevier; 2005.

17. Lima RBW, Figueiredo RJA, Andrade AKM, Duarte RM. Otimizando a Estética do Sorriso através de Coroa Cerâmica: Relato de caso. RBCS. 2013;17(2):165-70.

18. Gomes EA, Assunção WG, Rocha EP, Santos PH. Cerâmicas odontológicas: O estado atual. Cerâm. 2008;(54):319-25.
19. Carvalho, RLA; de Faria, JCB; de Carvalho, RF; Cruz, FLG; Goyata, FR. Indicações, adaptação marginal e longevidade clínica de sistemas cerâmicos livres de metal: uma revisão da literatura. Int J Dent. 2012;11(1):55-65.

20. Junior NC, Carvalho S. Metalocerâmica, IPS Empress I, II e IPS e.Max: uma revisão de literatura [Monografia] Lages: Instituto de Ciências da Saúde Funorte; 2011.

21. Zavanelli AC, Dekon SFC, Zavanelli RA, Mazaro JVQ, Nepomuceno VC, Fernandes AUR. Condicionamento gengival. PCL. 2004;6(32):357-63.

\section{CONFLITO DE INTERESSES}

Os autores declaram não haver conflitos de interesse.

\section{AUTOR PARA CORRESPONDÊNCIA}

Gisely Naura Venâncio

ginaura@gmail.com

Submetido em 22/04/2016 Aceito em 29/05/2016 